\title{
FACTORS AFFECTING THE IN-VITRO MATURATION OUTCOMES
}

Ismail Mahmoud Samy 1,2,3,4, (Facharzt, M.D. Ph.D),

1 Department of Obstetrics and Gynecology, King Fahd University Hospital,

King Faisel University, Dammam, KSA

2 Department of Obstetrics and Gynecology, Al Mana General Hospital, Khobar, KSA.

3 Department of Obstetrics and Gynecology, Faculty of Medicine,

Al Azhar University, Cairo, Egypt.

4 Department of Obstetrics and Gynecology, Campus Benjamin Franklin-

University Hospital, Homboldt University, Berlin, Germany

\section{ABSTRACT}

Objectives : In-vitro maturation of human oocytes is becoming increasingly important in treating some aspects of infertility. Our aim is to study the effect of concentration of recombinant follicle stimulating hormone(r-FSH) in culture media and follicle size, on oocytes maturation, fertilization, and cleavage and pregnancy rates in women with polycystic ovary syndrome.

Methods: In a randomized study of 100 women in an IVF program, 50 patients with 674 germinal vesicle oocytes were allocated to study the effect of three concentrations of r-FSH $(0.0,0.075$, and 7.5 IU) supplemented in culture media on oocyle maturation, fertilization, cleavage, and pregnancy rate. The remaining 50 patients produced $500 \mathrm{GV}$ and were allocated to siudy the effect of two follicle $r$ sizes $(8-10$ and $11-13 \mathrm{~mm}$ ) on the same parameters as above.

Results: Oocyte maturation rate significantly $(\mathrm{P}<0.05)$ increased from $47 \%$ at 0.0 (control) to 81 and $83 \%$ at 0.075 and $7.5 \mathrm{IU} / \mathrm{mI}$ of $r$-FSH concentration, respectively. Fertilization, cleavage, and clinical pregnancy rates showed a similar trend and significantly increased from 45 to 83 and $80 \%$; from 32 to 80 and $77 \%$ and from 0 to 17 and $14 \%$ at the three r-FSH concentrations, respectively. The results however showed that increasing r-FSH concentration to more than $0.075 \mathrm{IU}$ did not further improve the rates of the above parameters even when the concentration was increased 100 folds. Follicular size on the other hand showed significant $(\mathrm{P}<0.05)$ increase on the above parameters. Oocytes retrieved from $11-13 \mathrm{~mm}$ folicles showed higher rates of maturation, fertilization, cleavage, and pregnancy, than those retrieved from $8-10 \mathrm{~mm}$ follicles. The above parameters increased from 48 to $70 \%$ from 54 to $76 \%$ from 64 to $68 \%$, and from I to $22.5 \%$, respectively.

Conclusion: The results suggested that r-FSH supplementation in the culture media with a concentration of 0.075 IU was optimum in the present study and improving GV maturation, fertilization, cleavage and pregnancy rate. The results also showed that oocytes recovered from follicles with $11-13 \mathrm{~mm}$ in diameter provided higher maturation rates and a better subsequent developmental competence than those retrieved from smaller follicles of $8-10$ mm diameter.

Keywords; Follicles, IVM, ICSI, fertilization, pregnancy.

\section{INTRODUCTION}

Since the delivery of the first three children originating from in-vitro-matured oocytes obtained from ovarian biopsy specimens (1), research in
In-vitro maturation of human oocytes has shown significant progress and provided hope for certain groups of patients who have infertility rems. Human oocyte recovered from immature a cles, following retrieval can resume and complete ciosis 
in-vitro when cultured in media supplemented with r-FSH and hCG ${ }^{(2-5)}$. Published reports had shown that in-vitro matured oocytes could be fertilized, and result in pregnancy ${ }^{(6,2)}$ and birth of healthy babies (1.7). Despite the clinical utility of IVM in the field of human reproduction, its pregnancy and birth rates remain low compared to in-vitro matured oocytes. Nevertheless, IVM remained a low cost procedure and an optimum solution to some causes of infertility in certain groups of patients. Women with polycystic ovary syndrome have typical symptoms of abnormal endocrine parameters, hyperandrogenism, anovulation, numerous antral follicles in the ovary on ultrasound scan and infertility ${ }^{(2)}$. In PCOs patients the dominance of a particular follicle fails to occur and the cohort of the numerous growing follicles accumulate in the cortex ${ }^{(8)}$. These patients are extremely sensitive to exogenous gonadotropin when used for assisted reproduction and may develop ovarian hyper-stimulation, deep vein thrombosis (9) and ovarian cancer resulting from prolonged use of fertility drugs. There is now increasing interest to avoid these risks, by retrieving oocytes using minimal or no gonadotropin stimulation and then maturing them in-vitro in culture medium containing recombinant FSH, LH, or hCG. When oocytes are matured in-vitro, r-FSH or urinary gonadotrophins are added to the culture media to improve oocyte maturation in various studies (10). Recently both recombinant hCG and $L H$ in addition to FSH were used to achieve oocyte maturation (4), but without standardization of the optinum concentration which will yield the best results. This study is aimed at assessing the optimum range of r-FSH concentrations in culture media that produce the best maturation rate. In addition, since the follicle size is crucial in initiating the molecular cascade of normal nuclear and cytoplasmic maturation, our study is aimed to assess the effect of follicle size on oocyte maturation, fertilization and developmental competence.

\section{MATERIALS \& METHODS}

\section{Patients :}

Women with amenorrhea received the oral contraceptive Marvelon (Organon) once daily for 21-45 days to induce withdrawal bleeding at a specificed time to programme the cycle. A baseline vaginal ultrasound scan was performed for all women between day 1 and 2 of menstrual bleeding to ensure that no ovarian cysts were present. Ovarian stimulation with r-FSH $300 \mathrm{IU}$ is given daily for five days and continued as necessary. Transvaginal ultrasound scans were repeated on either cycle day 8 and/or the day of hCG administration to exclude the development of a dominant follicle. The size of all follicles on ultrasound scan had to be $<10 \mathrm{~mm}$ in diameter at every scan to proceed to oocyte retrieval, which was performed between days 8 and 10 of the cycle. All parients received $1000 \mathrm{LU}$ of hCG 36 hours before oocyte retricval.

\section{Oocyte retrieval and IVM Procedure :}

Transvaginal ultrasound guided oocyles collection was performed using a specially designed $17-\mathrm{G}$ single-lumen aspiration needle (Casmed, UK) with a reduced aspiration pressure of $7.5 \mathrm{kpa}$. Aspiration of all small follicles was performed under general anesthesia for all patients. Oocytes were collected in culture tubes containing warm Earl's balanced salt solutionn with $5000 \mathrm{IU} / \mathrm{ml}$ heparin. Immature oocytes were incubated in a culture dish containing $1 \mathrm{ml}$ of 3M (Medicult) medium supplemented wih r-FSH (puregon, Organon) and 5.00 IU/ml hCG (Pregnyl, Organon) at $37^{\circ} \mathrm{C}$ in an atomosphere of $5 \% \mathrm{CO} 2$ and $95 \%$ air with high humidity. After culture. the maturity of the oocyte was determined under the streo-microscope at 24 and 48 hours post collection. Oocytes were denuded of cumulus; maturity was determined by the presence of the first polar body. Suitable oocyles were injected with a single 
spermatozoon by micromanipulation (Research Instrument, UK). Following ICSI, each oocyte was transferred into $1 \mathrm{ml}$ of Medi-cult IVF medium in a tissue culture dish. Fertilization was assessed 18 hours after ICSI for the appearance of two distinct pronuclei and two polar bodies. Oocytes with two pronuclei were further cultured in Medi-cult IVF medium. Embryos were transferred on day 2 or 3 after ICSI.

\section{Endometrial priming :}

For endometrial preparation patients received estradiol valerate, Progynova (Schering Pharmaceutical, UK) starting on the day of oocyte retrieval, depending on the endometrial thickness on that day. If the endometrial thickness was $>4 \mathrm{~mm}$, a $6 \mathrm{mg}$ dose was given. Luteal support was provided by $100 \mathrm{mg}$ of progesterone (Gestone, Shire Pharmaceuticals, UK) once daily starting on the day of ICSI and continued, along with estradiol valerate until day 14 from the day of embryo transfer when a blood test for Beta hCG was done to ascertain pregnancy. If there is pregnancy the luteal support is continued till 12 weeks gestation.

The effect of FSH concentration in the culture media on in-vitro maturation of oocytes:

For this study, the patient group consisted of 50 women with PCO and they were all scheduled for ICSI. Patients were stimulated with 300 IU r-FSH (Puregon, Organon, Holland). The aim was to study the influence of follicle stimulating hormone concentration on the maturation, fertilization, cleavage and pregnancy. Recombinant FSH was added in three concentrations: $0.00,0.75,7.5 \mathrm{IU} / \mathrm{ml}$.

This experiment was performed on patients producing more than $15 \mathrm{GV}$ oocytes. Immediately after oocyte collection, the oocyles were equally aliquot in three groups of 5 oocytes each in three center-well dish containing $3 \mathrm{ml}$ Ham's Flo. Two
r-FSH concentrations were added in all two dishes and none as control, respectively. Oocyte maturation were then assessed 30 hours after incubation.

The effect of follicular size on the rate of oocyte maturation :

In this experiment, $50 \mathrm{PCO}$ patients were stimulated with $300 \mathrm{IU}$ (Purgeon, Organon, Holland) for 5 days, starting on day 2 of the menstrual cycle until day 6 when the transvaginal ultrasound scan was performed. The scan showed follicular size ranging between $8-13 \mathrm{~mm}$. The PCO patients were divided in two groups according to follicular size on the day of hCG, 8-1 and 11-13 mm. The oocytes from these patients were incubated for 30 hours in Ham's F10 supplemented with $0.075 \mathrm{IU} \mathrm{r}-\mathrm{FSH} / \mathrm{ml}$. Oocyte maturation, fertilization, cleavage, and pregnancy rates were then assessed.

\section{RESULTS}

The results in the present study were based on data generated from two experiments. The first experiment was designed to define the optimum r-FSH concentration. Our data showed that 0.075 $\mathrm{IU} / \mathrm{ml}$ was optimum and provided higher maturation, fertilization and pregnancy rates than $7.5 \mathrm{IU} / \mathrm{ml}$ a the control. Based on this optimum concentration a second experiment was designed to study the effect of follicle size on oocyte maturation, fertilization and developmental competence. Details regarding number of oocytes coollected, maturation, fertilization and pregnancy rates after in vitro maturationin media containing different concentrations of r-FSH are shown in table I. The above parameters resulting from two different follicle sizes are shown in table II. Recombinant FSH concentration had significantly $(p<0.05)$ increased rate of oocytes maturation from $47 \%$ at $0 \mathrm{IU}$ to 81 and $83 \%$ at 0.075 and $7.5 \mathrm{IU} / \mathrm{ml}$, respectively. 
Fertilization, cleavage, and clinical pregnancy rates showed similar trends and significantly increased from 45 to 83 and $80 \%$; from 32 to 80 and $77 \%$ and from 0 to 17 and $14 \%$ at the three concentrations, respectively. The results however showed that increasing $r$-FSH concentration for more than 0.075 IU did not further improve maturation, fertilization. cleavage and pregnancy rates even when the concentration was increased 100 folds. Follicular size on the other hand showed significant $(\mathrm{P}<0.05)$ effect on the above parameters. Oocytes retrieved from 11-13 $\mathrm{mm}$ follicles showed higher rates of maturationnm, fertilization an pregnancy, than those retrieved from $8-10 \mathrm{~mm}$ follicles. The above paramelers increased from 48 to $70 \%$; from 54 to $76 \%$ and from 11 to $22.5 \%$ in the two follicle sizes, respectively. In the firs. group study, the 6 and 5 pregnancies resulting from oocyte cultured in media containing 0.075 and $7.5 \mathrm{IU} / \mathrm{ml}$ all ended in delivery of healthy children. On the other hand, in the second group study, the two pregnancies resulting from 8-10 $\mathrm{mm}$ follicles size completed full term, whereas two of the nine pregnancies in the $10-12 \mathrm{~mm}$ follicle size group ended in miscarriage and the remaining seven pregnancies ended in delivery of healthy babies.

\section{DISCUSSION}

There are various factors involved in oocyte in-vitro maturation: most important among these are the exposure of immature oocyles to gonadotrophin in culture media and the follicle size from which the oocytes were retrieved. Recombinant FSH, LH and $\mathrm{hCG}^{(8)}$, and puritied gonadotropin ${ }^{(9)}$ were used to induce oocyte maturation in vitro. The present study showed that in-vitro matured oocytes from PCOS patierts had the potential to undergo successful maturation, fertilization and the resultant embryos showed good cevelopmental competence. Following the IVM procedure, embryo transfer culminated in clinical pregnancies and birth of healthy children. All the oocyles retrieved in this study were at Germinal vesicle (GV) stage. The latter were defined as germinal vesicle stages (GV) which represent oocytes arrested at prophase of meiosis - 1 with prominent discemable germinal vesicle nucleus. In the present study in-vitro maturation rates of oocytes cultured in media containing $0.075 \mathrm{rU} / \mathrm{ml}$ was $81 \%$ which is higher when compared with $62 \%(12), 67 \%(13)$, $71 \%^{(6)}, 61 \%^{(14)}, 66 \%^{(15)}, 58 \%^{(16)}$, and $55.9 \%^{(4)}$. The variations in maturation rates between the present study and those mentioned above may be due to the composition of the culture medium used and protein supplements. In the present study, our optimum culute time (30h) was comparable with other studies (16). Inadequacics of cultured media can not be ruled out as a posstble cause for low IVM success (15). There is evidence to suggest that culture media used for IVM adequately support nuclear maturation. but failed to produce oocytes with cytoplasmic maturation. While we used synthetic serum supplement as a source of proteins, in other studies fetal bovine serum (FBS) was used. FBS was considered more crucial for bovine oocyte maturation than human. In addition, our base medium was Hams F10 which was designed to meet the nutritional and maturational needs for human oocytes. Other reasons for the low maturation, fertilization and the developmental competence may be due to polycystic ovary syndrome as the main cause of infertility. Recent reports had shown that the low maturation rates in IVM can be improved by priming patients with gonadotrophins. Patient priming with -FSH and hCG before retrieval may have contributed to the increase of the maturation rate in our study, trounson and colleagues $(2001)^{(2)}$ reported a higher maturation rate $(71 \%)$ in FSH treated women when compared wh untreated women (44\%). Folliele priming with r-FSH and hCG before oocyce retrieval had significantly increased rate and speed of oocyte 
maturation ${ }^{(3)}$. It was found that $75 \%$ of oocyces recovered from super ovulated and primed patiens reached metaphase II after 30 of of culure while the same percentage was reached after $42-45$ h of culture. Despite the relatively high maturation, fertization and cleavage rates shown in the present study, our pregnancy rate remained relatively low (22.5\%) compared $1027 \%^{(16)}$, and $40 \%$ (2). The low pregnancy rate shown in IVM cases in general is partly attributed to abnormality during cytoplasmic maturation ${ }^{(5)}$. However pregnancy in other studies reached even higher rates $(40 \%)$ following administration of 10000 IU hCG before immature oocyte retricval $(2,17)$. In the present study, all patients were primed with 5000 IU hCG rather than 10000 IU to reduce the risk of OHSS, and this might have contributed to the low pregnancy rate According to Chian et al. (1999), Son et al., (2002) and Hreinsson et al., (2003) $(1,18,4)$, hCG priming improved the percentage of oocytes achieving maturation and hastens the maturation process. The overall oocyte quality might be reduced by the oocyte retrieved from PCOS due to high androgen level. although hCG priming improved maturation rate of immature oocytes ${ }^{(17)}$, there is no evidence to suggest that FSH priming has the similar effect on pregnancy rates except for few exceptional cases ${ }^{(10)}$. In other studies FSH priming made no difference to oocyte recovery, maturational and developmental potential, fertilization rate and pregnancy rate $(3,4)$.

The effect of follicle size on oocyte maturation, fertilization, cleavage, and pregnancy rates showed some interesting results. Our data showed that oocyte maturation rate and developmental competence have significantly increased with increase in follicle sizes. Eppig et al., 1992 concluded also that developmental competence of oocytes depends on the follicle and oocytes size ${ }^{(10)}$. The growth in size is due to the fact that oocytes synthesize and store mRNA and proteins which are essential for the completion of maturation and for the subsequent acquisition of embryo development $(11)$. This probably explains the relatively higher maturation, fertilization, cleavage, and pregnancy rate in oocytes obtained from follicles with $11.13 \mathrm{~mm}$ sice rather than $8.10 \mathrm{~mm}$. Ii has been found that the capacity for meiotic competence in animals such as rhesus monkey ${ }^{(12)}$ increases with follicle size and not strictly correlated with maximum oocyte diameter or formation of antrum. Conversely, human oocytes restme maturation at low rate compared with other species ${ }^{(13)}$. This may be due to the fact that meiotic competence occurs late during the growth phase or maturation need both stimulation and removal of inhibition ${ }^{(14)}$. This also indicates that human oocytes have a size-dependant ability to resume meiosis and complete maturation in-vitro when oocyte diameter increases from 90 to $120 \mu \mathrm{m}$ Moreover, meiotic competence was found to depend on the size of the follicle and the stage of the menstrual cycle. Human oocytes retrieved from follicles of $9-15 \mathrm{~mm}$ in diameter complete meiotic maturation to metaphase II at a higher rate than oocytes from follicles of $3-4 \mathrm{~mm}$ in diameter ${ }^{(4)}$. However the minimum follicle diameter from which human oocytes would mature was reported to be 5 $\mathrm{mm}^{(15)}$. The effect of follicle size on oocyte maturation, fertilization and subsequent developmental competence has been reported in various other studies ${ }^{2)}$. These reports reached to the same conclusion as in our present study. Simonelli ct al. (1985) found that human oocytes maturation rate significantly increases with follicular size after super ovulation with human menopausal gonadotrophin (hMG) and Human chorionic gonadotrophin ${ }^{\text {(16) }}$

Scott et al., 1989. Found that the proportion of oocytes maturation increased from $9 \%$ to $30 \%$ when oocytes were obtained from two follicular sizes of $<11$ and $12-14 \mathrm{~mm}$, respectively ${ }^{(17)}$. Follicle size was also reported to affect the fertilization rate. Recent studies had shown rather lower rate of oocyte 
maturation for those retrieved from smaller follicles (58\%) compared to those retrieved from larger follicles $(89 \%)^{(18)}$. The higher maturation rate in their study may be due to the fact that larger follicles may produce larger oocyles at metaphase one stage which may have better maturation capacity/potential than germinal vesicle oocytes. According to Trounson et al., (2001) oocyte size is directly related to maturation and subsequent embryo development ${ }^{(2)}$. This may be due to molecular cascade of both cytoplasmic and nuclear maturaiton as initiated when the oocytes reach certain size. Following gonadotrophin stimulation, it was found that fertilization rate was lower in oocytes obtained from follicles $<10 \mathrm{~mm}$ than those obtained from larger follicles $(19,20,21)$. The same report found that cleavage rate was also reduced with follicular size. Combelles et al., (2002) attributed the low efficacy of IVM to intrinsic differences in oocytes which results in developmental capacity variation due to incomplete or abnormal oocyte growth (22). It has also been suggested that failure in developmental competence may be due to aberrations in cytoplasmic maturation (23). At the molecular level, meiotic competence in oocytes has been related to germinal vesicle chromatin organization, and meiotic cell cycle status. While in the present study pregnancy rate increased with follicle size. Other reports found that implantation, pregnancy, and birth rates were independent of follicular size(19,21) . Our data suggested that when oocytes were cultured in IVM media containing $0.075 \mathrm{IU} \mathrm{r}$-FSH or retrieved from follicle size exceeding $10 \mathrm{~mm}$, a comparable or even better maturation rates and developmental competence than results in many recent reports were obtained. The results also showed that the above parameters were lower when the oocytes were retrieved from ollicles smaller than $10 \mathrm{~mm}$.

In the present study FSH priming however had increased E2 level, but it did not show any increase in endometrial thickness or in any of the parameters studied previously. Similar conclusion was also reported in several other studies $(24,6)$. Thickness of the endometrium is directly associated with pregnancy rates. According to Gonsen and Casper 1990, endometrial thickness of $8.6 \mathrm{~mm}$ was found capable of achieving pregnancy (11). Our study showed a mean endometrial thickness of $8.2 \mathrm{~mm}$ and all patients showed triple line ring pattern on the day of embryo transfer. In our study, the endometrial thickness on the day of hCG was better than other studies which achieved higher pregnancy rates. Lin et al., reported $31 \%$ pregnancy rate in FSH primed patient with mean endometrial thickness of $8.2 \mathrm{~mm}$ on the day of hCG injection ${ }^{(6)}$. Poor endometrial thickness was partly responsible for reduction in pregnancy rate in IVM cycles. It was reported that endocrinology in IVM patients is disrupted after oocyte retrieval and may result in FSH and $\mathrm{LH}$ rise and E2 drop (25). Inadequate E2 level may be harmful to endometral development and may reduce pregnancy rate. Another reason for reduced pregnancy rate in IVM cycles may be due to chromosomal anomalies. While some studies had shown an incidence of $78.5 \%$ chromosomal anomalies in in-vitro matured oocytes from stimulated cycles ${ }^{(26)}$; others reported $20 \%(27)$ Further research is required to assess the chromosomal anomalies in in-vitro matured oocytes. Reduced pregnancy rate in our IVM cycle may be related to low number of embryos transferred. In the present study an average of 2.3 embryos per patient were transferred. Studies had shown that implantation rates of IVM were relatively low compared to IVF cycle and to overcome such problem more embryos were transferred in IVM cycles $(7,35,36,37)$. In our study, $80 \%$ of cases, only 1.7 embryos were transferred. This may have contributed to the low pregnancy rate achieved in the present study. Anesthesia, such as propofol was found detrimental 
to in-vitro maturation, fertilization and cleavage in mouse oocytes. In this study we used the same anestheric agent which may have similar effect although this has not been proved in human oocytes and more research is required ${ }^{(35-37)}$. In the present study, factors affecting immature oocyte maturation and developmental competence are not fully explored. There are many gaps that need to be bridged and other factors need to be closely investigated. The effect of growth hormone in culture media during oocyle maturation, chromosomal anomalies as well as the effec of anesthesia are both worhtwhile to be investigated thoroughly.

\section{ACKNOWLEDGMENT}

Many thanks to Mr. Murthy P.S.R. IVF unit, Al Mana General Hospital, Dammam, KSA for his great effeorts as the chicf embryologist of the unit.

Table I : Effect of recombinant FSH concentration on oocyte maturation, fertilization, embryo cleavage and clinical pregnancy.

\begin{tabular}{|l|l|l|l|}
\hline \multirow{2}{*}{ Parameters } & \multicolumn{3}{c|}{ r-FSH Concentrations (IU) } \\
\cline { 2 - 4 } & 0.00 & 0.075 & 7.5 \\
\hline GV collected & 225 & 219 & 230 \\
\hline Matured oocyte & $105(47 \%)^{\mathrm{a}}$ & $178(81 \%)^{\mathrm{b}}$ & $186(83 \%)^{\mathrm{b}}$ \\
\hline Fertilized oocyte & $47(45 \%)^{\mathrm{a}}$ & $147(83 \%)^{\mathrm{b}}$ & $149(80 \%)^{\mathrm{b}}$ \\
\hline Cleaved embryos & $15(3 \%)^{\mathrm{a}}$ & $119(80 \%)^{\mathrm{b}}$ & $116(77 \%)^{\mathrm{b}}$ \\
\hline Transferreed embryos & $12(2 / \mathrm{ET})$ & $55(1.4 \mathrm{ET})$ & $60(1.7 / \mathrm{ET})$ \\
\hline Clinical Pregnancy & $0.00^{\mathrm{a}}$ & $6(17 \%)^{\mathrm{b}}$ & $5(14 \%)^{\mathrm{b}}$ \\
\hline No ET & 6 & 39 & 35 \\
\hline
\end{tabular}

N.B Data with similar superscripts letters are not significantly $(p>0.05)$ different .

Table II : Effect of follicular size on oocyte maturation, fertilization, embryo cleavage, and clinical pregnancy.

\begin{tabular}{|l|l|l|}
\hline \multirow{2}{*}{ Parameters } & \multicolumn{2}{c|}{ Follicular size (mm) } \\
\cline { 2 - 3 } & $8-10$ & $11-13$ \\
\hline GV collected & 250 & 250 \\
\hline Matured oocyte & $120(48 \%)^{\mathrm{a}}$ & $177(70 \%)^{\mathrm{b}}$ \\
\hline Fertilized oocyte & $65(54 \%)^{\mathrm{a}}$ & $138(76 \%)^{\mathrm{b}}$ \\
\hline Cleaved embryos & $42(64 \%)^{\mathrm{a}}$ & $094(68 \%)^{\mathrm{b}}$ \\
\hline Transferreed embryos & $33(1.9 / \mathrm{ET})$ & $060(1.7)$ \\
\hline Clinical Pregnancy & $2(11 \%)^{\mathrm{a}}$ & $09(22.5 \%)^{\mathrm{b}}$ \\
\hline No ET & 17 & 40 \\
\hline
\end{tabular}

N.B Data with similar superscripts letters are not significantly $(p>0.05)$ different 


\section{REFERENCES}

1. Chian R.C., Buckett, W.M., Too, L.L., and Tan, S.L. Pregnancies resulting from in vitro matured oocytes retrieved from patients with polycystic ovary syndrome after priming with human chorionic gonadotropin. Fetil. And Steril., 1999; 72: 639-642.

2. Trounson, A., Anderie, C., and Jone, G. Maturation of human oocytes in vitro and their developmental competence. Reproduction, 2001; 121:51-75.

3. Steward, J. A., Hamilton, P. J., and Murdoch, A. P. Thromboembolic disease associated with ovarian patients' stimulation and assisted conception techniques. Hum. Reprod. 1997; 12: 2167-2173.

4. Whitacre, K.S., Seifer, D.B., Friedman, C.I., Coskun, S., Kennard, K.A., Kim, M.H., and Alak B. M. Effects of ovarian source, patient age and menstrual cycle phase on in vitro maturation of immature human oocytes Fertil. and Steril., 1998, 70, 1015-1021.

5. Hreinsson, J., Rosenlund, B., Friden, B., Levkov, L., Ek, I., Sikkari, A., Hovata, O., and Fridstrom. Recombinant $\mathrm{LH}$ is equally effective as recombinant hCG in promoting oocyte maturation in a clinical in-vitro maturation progmame: a randomized study. Hum, Reprod., 2003; 18: 2131.

6. Lin, Y., Hwang, J., Huang, L., Mu, S., Seow, K., Chung, J., Hsieh, B., Huang, S., Chen, C., and Chen, P. Comination of $\mathrm{FSH}$ priming and $\mathrm{hCG}$ priming for in-vitro maturation of human oocytes. Hum. Reprod $.2003 ; 18: 1632$.

7. Cha, K. Y., Koo, J. J., Ko, J. J., Choi, D. H., Han, S. Y., and Yoon, T. K. Pregnancy after in vitro fertilzation of human follicular oocytes collected from nonstimulated cycles, their culture in vitro and their transfer in a donor oocyte program. Fertil., and Steril., 1991; 55: 109-113.

8. Chian, R., C., Gulekli, B., Buckett, W. M. and Tan, S. L. Pregnancy and delivery after cryopreservation of zygote produced by in-vitro matured oocytes retrieved from a women with polycystic ovarian ovarian syndrome, Hum. Reprod. 2001; 16: 1700-1702.

9. Jaroudi, K. A., Hollanders, J. M. G., Elnour, A. M., Roca G. L., Atared, A. M. and Coskun, S. Embryo development and pregnancies from in vitro matured and fertilized human oocytes. Hum. Reprod., 1999; 14: 1749-1751.

10. Eppig, J. J., Wigglesworth, K., and O'Brien, M. J. Developmental capacity of mouse oocyles matured in vitro: effects of gonadotropic stimulation, follicular origin and oocyte size. Jounal of Reproduction and Fertility, 1992; 95, 119-127.

11. Gonsen, Y, and Casper, R. F. Prediction of implantation by the sonographic appearance of the endometrium during controlled ovarian stimulation for in vitro for in-0vitro fertilization (IVF). J. In Vitro Fertilizaion, embryo Transfer. 1990; 7 146-152.

12. Schramm, R. D., Tennier, M. T., Boatman, D. E. and Bavster, B. D. Chromatin configurations and meiotic competence of oocytes are related to follicular diameter in non-stimulated rhesus monkeys. Boil of Reprod. 1993; 48. 349-356.

13. Edwards, R. G. Maturation in viro of mouse, sheep, cow, pig, rhesus monkey and human ovarian oocytes. Nature, 1965; 208, 349-351.

14. Lefevre, B., Gougeon, A. and Testart, J. In vitro oocyte maturation: Some questions concerning the initiation and prevention of this process in humans. Fum. Reprod. 1987; 2, 495-497.

15. Wynn, P., Picton, H. M., Krapez, J. A., rutherford, A. J., Balen, A. H., and Gosden, R. G. Pretreatment with follicle stimulating hormone promotes the numbers of human oocytes reaching metaphase II by in vitro maturation. Hum. Reprod. 1998; 13, $3132-3138$

16. Simonetti, S., Veeck, L. L., and Jones. H. W. Correlation of follicular fluid volume with oocyte morphology from follicles stimulated by human 
menopausal gonadotropin. Fertili and Steril., 1985; 44, 177-180.

17. Scott, R. T., Hofman, G. E., Muasher, S. J., Acosta, A. A., Kreiner, D. K., and Rosenwaks, Z. Correlation of follicular diameter with oocyte recovery and maturity at the time of transvaginal follicular aspiration. Journal of In Vitro Fertilization and Embryo Transfer. 1989; 6, 73-75.

18. Gaspard, O., Godin, P. A., Thonon, F., Jouan, C., Hincourt, N., Ravet, S., Wijzen, F., Dubois, M., and Foidart, J. M. Outcome of oocytes derived from large and small follicles in hyperstimulated cycle. (Abstract). (2003; the $19^{\text {th }}$ Annual Meeting of the ESHRE, Madrid.

19. Wittmaack, F. M., Kreger, D. O., Blasco, L., Tureck, R. W., Mastroianni L., Jr. and Lessey, B. A. Effect of follicular size on oocyte retrieval, fertilization cleavage and embryo quality in in-vitro fertilization cycles: a 6-year data collection. Fertil. and Steril. 1994; 62, 1206-1210.

20. Dubey, A. K., Wang, H. A., Duffy, P. and Penzias, A. S. The correlation between follicular measurements oocyte morphology and fertilization rates in an in vitro fertilization program. Fertil and Steril., 1995, 64, 787-790.

21. Salha, O., Abusheika, N., and Sharma, V. Dynamics of human follicular growth and in vitro oocyte maturation. Hum. Reprod. Update. 1999; 4, 816-832.

22. Combelles, C. M., Ceklleniak, N. A., Racowsky, C., an Albertini, D. F. Assessment of nuclear and cytoplasmic maturation in-vitro maturedd human oocytes. Hum. Reprod., 2002; 17: 1006-1016.

23. Moor, R. M., Dai, Y., Lee, C., and Fulka J., Jr, (1998) Oocyte maturation and embryonic failure. Hum. Reprod. Update. 4, 223-236.

24. Mikkelsen, A. L., and Lindenberg, S. Benefit of FSH priming of women with PCOS to the in-vitro maturation procedure and the outcome: a randomized prospective study. Reproduction, 2001; 122: $587-592$
25. Requena, A., Neuspllier, F., Cobo, A. C., Aragones, M., garcia-Ve asco, J. A., Remohi, J., Simon, C., and Pellicer, A. Endocrinological and ultrasonographic variations after immature oocyte retrieval in a natural cycle. Hum Reprod., 2001; 16: 1833-1837.

26. Nogueira, D., staessen, C., Van de Velde, V., and Van Steirteghen, A., Nuclear status and cytogenetics of embryos delivered from in-vitro matured oocytes. Fertil. Steril. 2000; 74: 295-298.

27. Gras, L., McBain, J., Trounson, A., and Kola. 1. The incidence of chromosome aneuploidies in stimulated and unstimulated (natureal) uniseminated human oocytes. Hum. Reprod., 1992; 7: 1396-1401.

28. Cha, K. Y., and Chian R. C. Maturation in vitro of immature human oocytes for clinical use. Hum. Reprod. Update. 1998; 4: 03-120.

29. Almahbobi A. and Trounson A. O. the role of intraovarian regulators in the aetiology of polycystic ovarian syndrome. Reproductive Medicine Review, 1996; 5, 151-168.

30. Anderiesz, C. and Trounson A. O. The effect of testosterone on the maturation and developmental capacity of murine oocytes in vitro Hum. Reprod., $1995 ; 10,2377-2381$.

31. Barnes, F. L., Kausche, A., Tiglias, J., Wood, C., Wilton, L. and Trounson, A., Production of embryos from in vitro-matured primary human oocytes. Fertil and Steril., 1996; 65, 11561-1156.

32. Child, T., J., Abdul-Jalil, A. K., gulekli, B., and Tan. S. L., in vitro maturation and fertilization of oocytes from unstimulated normal ovaries. polycystic ovaries, and women with polycystic ovary syndrome. Fertil Steril., 2001 ; 76, 936-942.

33. Goudet, G., Bezard, J., Duchamp, G. Gerard, N.. and Palmer, E. Equine oocyte competence lor unclear and cytoplasmic in vitro maturation: effect of follicle size and hormonal environment. Biol. of Reprod. 1997; 57, 232-245.

34. Hwu, Y. M., Lee, R. K., Chen, C. P., Su, J. T., Chen, Y. W., and Lin, S. P., Developmental of hatching 
blastocyts from immature human oocytes following in vitro maturation and fertilization using a co-culture system. HGuman reprod., 1998; 13, 1916-1921.

35. Martino, A., Magos, T., Palomo, M. J., and Paramio, M. T. Meiotic competence of prepubertal goat oocytes. Theriogenology, 1994; 41, 969-980.

346. Motlik, J., and Fulka, J. Factors affecting meiotic competence of pig Oocytes. Theriogenology, 1986; $25,87-96$.

37. Son, W. Y., Yoon, S. H., Lee, S. W., Ko, Y., Yoon, H. G., and Lim, J. H., Blasocyst development and pregnancies after IVF of mature oocytes retrieved from unstimulated patients with PCOS after in-vivo hCG priming: Case r. Hum. Reprod., 2002; 17 , 134-136. 